\title{
REMOVABLE SINGULARITIES FOR $n$-HARMONIC FUNCTIONS AND HARDY CLASSES IN POLYDISCS
}

\author{
DAVID SINGMAN
}

\begin{abstract}
Let $\phi$ be any strongly convex function. For an open subset $G$ of a polydisc $U^{n}$ the Hardy class $H_{\phi}(G)$ is the set of analytic functions $f$ on $G$ for which $\phi \circ \log |f|$ has an $n$-harmonic majorant. It is shown that $H_{\phi}\left(U^{n} \backslash E\right)=H_{\phi}\left(U^{n}\right)$ for any relatively closed $n$-negligible subset $E$ of $U^{n}$.
\end{abstract}

1. Let $U^{n}$ be the open unit polydisc in $\mathbf{C}^{n}$, and let $E$ be a closed subset of $U^{n}$. Consider the analytic functions $f$ on $U^{n} \backslash E$ such that, for some strongly convex $\phi$, $\phi \circ \log |f|$ has an $n$-harmonic majorant on $U^{n} \backslash E$. Denote this set by $H_{\phi}\left(U^{n} \backslash E\right)$. With various conditions on $E$, the problem of showing $H_{\phi}\left(U^{n} \backslash E\right)=H_{\phi}\left(U^{n}\right)$ has been recently considered. Taking the special case of $\phi(t)=e^{p t}$, Cima and Graham [3, Theorem B] solved the problem for $E$ an analytic subvariety of $\mathbf{C}^{n}$. This was improved by Järvi [6, Theorem 2] for $E n$-polar. The purpose of this note is to extend the above results by taking $E$ to be $n$-negligible [9, p. 32]. This result has been proved by Cegrell [2] by entirely different means. To us, the proof we present here seems simpler and more direct. It makes systematic use of a general theorem on removable singularities for $n$-harmonic functions, Theorem 1 below. Our proof also works for any strongly convex $\phi$.

The author would like to thank K. Gowrisankaran and I. Graham for discussions related to this work.

2. Let $\Omega$ be a Brelot harmonic space with positive potential and a countable base for open sets [1]. Assume also constants are superharmonic. We restrict our attention now to an open subset $U$ of $\Omega$. Following Parreau [7] we call a nonnegative harmonic function quasi-bounded if it is the limit of a nondecreasing sequence of bounded harmonic functions, and singular if it majorizes no positive, bounded harmonic function. Then, using precisely the same argument as in, Heins [5, p. 7], where he considered harmonic functions on Riemann surfaces, we can show every positive harmonic function $u$ can be decomposed uniquely as a sum of a quasi-bounded and a singular harmonic function $u=q+s$. Furthermore, $u \rightarrow q, u \rightarrow s$ are both positively homogeneous and additive. Hence $u$ quasi-bounded or singular implies any

Received by the editors April 18, 1983.

1980 Mathematics Subject Classification. Primary 32D20, 32A35; Secondary 31D05.

Key words and phrases. Polydisc, $n$-superharmonic function, $H_{\phi}$ class, Brelot space, $n$-negligible set. 
positive harmonic minorant of $u$ has the same property. Again, as in Heins [5, p. 18], we can prove the following

LEMma. Let $U \subset \Omega$ be open, $u$ subharmonic on $U$ and $\phi:[-\infty, \infty) \rightarrow[0, \infty)$ strongly convex. If $\phi \circ u$ has a superharmonic majorant on $U$, then its least harmonic majorant is quasi-bounded.

COROLlary. If $U=\Omega \backslash P$, where $P$ is polar, then $u$ has a unique subharmonic extension to $\Omega$.

Indeed, since $x \leqslant \phi(x)+c$ for some $c$ and all $x, u$ has a harmonic, quasi-bounded majorant $h$ on $\Omega \backslash P$. Thus there is a nondecreasing sequence $h_{n}$ of nonnegative, bounded harmonic functions on $\Omega \backslash P$ converging to $h$. Since $P$ is polar, each $h_{n}$ extends harmonically across $P[\mathbf{1}$, p. 125] and by Axiom 3 the limit is harmonic. Thus $u$ is locally bounded near $P$ and hence has a unique subharmonic extension to $\Omega[1$, p. 125]. This proves the result.

3. Consider now $\Omega_{1}, \Omega_{2}$ Brelot spaces as above. Put $\Omega=\Omega_{1} \times \Omega_{2}$. A function is 2 -harmonic on $\Omega$ if it is continuous and separately harmonic. The 2 -subharmonic and 2-superharmonic functions are defined similarly [4]. We remark that we consider 2 spaces only for the sake of convenience. All the following have obvious analogues for any finite product.

In this setting the Harnack Theorem concerning the limit of an increasing sequence of 2-harmonic functions is valid [4, p. 31]. The existence of the least 2-harmonic majorant on $\Omega$ for a 2-subharmonic function with a 2-superharmonic majorant is also valid [4, p. 40]. It follows using a similar proof that the Lemma is valid for this space and this 2-harmonic structure if we take $U$ to be $\Omega$. For general $U$ the proof breaks down as we no longer have least 2-harmonic majorants.

We wish now to prove an analogue of the corollary. For this purpose the exceptional set we consider analogous to the polar set is the 2-negligible set. A set $E$ is 2-negligible if there are polar sets $P_{i}$ in $\Omega_{i}, i=1,2$, such that for all $x$ in $\Omega_{1} \backslash P_{1}$ the section $E_{x}=\left\{y \in \Omega_{2} \mid(x, y) \in E\right\}$ is polar in $\Omega_{2}$ and for all $y$ in $\Omega_{2} \backslash P_{2}$ the section $E^{y}=\left\{x \in \Omega_{1} \mid(x, y) \in E\right\}$ is polar in $\Omega_{1}$ [9]. This is more general then the sets on which 2-superharmonic functions take the value $+\infty$, the so-called 2-polar sets. For $\Omega$ the unit polydisc this is exactly the exceptional set considered by Cegrell in [2]. (For $n>2$ the $n$-negligible sets are defined inductively in the obvious way.) We have the following theorem on removable singularities.

TheOREM 1 [9, TheOREM 4.9]. Let $E$ be a closed 2-negligible set and $v$ a 2-superharmonic function on $\Omega \backslash E$ that is locally lower bounded near $E$. Then there is a unique 2-superharmonic function $w$ on $\Omega$ such that $w=v$ on $\Omega \backslash E$. It is given by $\liminf _{y \rightarrow x} v(y), y$ in $\Omega \backslash E$.

We have the following generalization of the Corollary.

Proposition. Let $E$ be a closed 2-negligible set. Let $u$ be 2-subharmonic on $\Omega \backslash E$ such that $\phi \circ u$ has a 2-superharmonic majorant $v$ on $\Omega \backslash E$. Then $u$ extends 2-subharmonically across $E$ and $\phi \circ u$ has a quasi-bounded 2-harmonic majorant on $\Omega$. 
Proof. We may assume $u$ is nonnegative. Indeed $u^{+}$is 2-subharmonic, $u \leqslant u^{+}$ and $\phi \circ u^{+} \leqslant \phi \circ u+\phi(0)$ so $\phi \circ u^{+}$has a 2 -superharmonic majorant on $\Omega \backslash E$. We may also assume, by Theorem 1, that $v$ is defined and 2-superharmonic on $\Omega$.

Let $P$ be a $G_{\delta}$ polar subset of $\Omega_{1}$ such that for all $z$ in $\Omega_{1} \backslash P, E_{z}$ is polar in $\Omega_{2}$ and $v(z, \cdot)$ is not identically $+\infty$ on a connected component of $\Omega_{2} \backslash E_{z}$. Define $W$ to be the points $(x, y)$ of $\Omega \backslash E$ such that $v(\cdot, y)$ is not identically $+\infty$ on a connected component of $\Omega_{1} \backslash E^{y}$ and $E^{y}$ is polar. Then $\Omega \backslash W$ is clearly 2-negligible. Let now $\omega, \delta$ be regular domains in $\Omega_{1}, \Omega_{2}$, respectively. Since products of harmonic measures do not charge 2-negligible sets and $u$ is majorized by $v$, it follows that $\iint u d\left(\rho_{x}^{\omega} \times \rho_{y}^{\delta}\right)$ is a 2-harmonic function of $(x, y)$ on $\omega \times \delta$. Notice that the integration is performed on $\Omega \backslash E$. We show that this function majorizes $u$ on $\omega \times \delta \backslash E$. By Theorem 1 we only have to verify this for $(x, y)$ in $W$.

$$
\begin{aligned}
\iint u d\left(\rho_{x}^{\omega} \times \rho_{y}^{\delta}\right) & =\int d \rho_{x}^{\omega}(z) \int u\left(z, z^{1}\right) d \rho_{y}^{\delta}\left(z^{1}\right) \\
& \geqslant \int u(z, y) d \rho_{x}^{\omega}(z) .
\end{aligned}
$$

Indeed, since $\rho_{x}^{\omega}(P)=0$, we may assume $z$ is not in $P$ so $E_{z}$ is polar in $\Omega_{2}$. Thus $u(z, \cdot)$ is subharmonic and $\phi \circ u(z, \cdot)$ is majorized by the superharmonic $v(z, \cdot)$, so, by the Corollary, $u(z, \cdot)$ has a unique subharmonic extension across $E_{z}$. Inequality (1) follows since $z$ can be assumed outside of $E^{y}$. For similar reasons we have

$$
\int u(z, y) d \rho_{x}^{\omega}(z) \geqslant u(x, y) \text {. }
$$

This shows $u$ is locally upper bounded near $E$. Theorem 1 then implies it extends 2-subharmonically across $E$ and $\phi \circ u$ is majorized by $v$ on $\Omega$. Finally, $\phi \circ u$ has a quasi-bounded 2-harmonic majorant by the remarks at the beginning of $\S 3$. The proof is complete.

4. We can now prove the following theorem on Hardy classes of analytic functions on $D$.

THEOREM 2. Let $E$ be a closed, 2-negligible subset of $U^{2}$. Let $f$ be analytic on $U^{2} \backslash E$ such that, for some strongly convex $\phi, \phi \circ \log |f|$ has a 2-superharmonic majorant on $U^{2} \backslash E$. Then there is an analytic $F$ on $U^{2}$ such that $F=f$ on $U^{2} \backslash E$ and $\phi \circ \log |F|$ has a 2-harmonic majorant on $U^{2}$.

Proof. We assume $f$ is not identically zero. Then $\log |f|$ is 2-subharmonic on $U^{2} \backslash E$ and $\phi \circ \log |f|$ has a 2-superharmonic majorant on $U^{2} \backslash E$. By the Proposition, $\log |f|$ extends 2-subharmonically across $E$ and $\phi \circ \log |f|$ has a 2-harmonic majorant on $U^{2}$. It follows that the real and imaginary parts $g, h$ of $f$ are locally bounded near $E$, so by Theorem 1 they have unique 2-harmonic extensions $G, H$ across $E$. Since the partial derivatives of $G, H$ are 2-harmonic and the CauchyRiemann equations for $F=G+i H$ are valid in $U^{2} \backslash E$, they are also valid on $E$ by Theorem 1. Finally, $\phi \circ \log |F|$ must agree with the extension of $\phi \circ \log |f|$ again by Theorem 1. This completes the proof. 


\section{REFERENCES}

1. M. Brelot, Lectures on potential theory, Lectures on Math., 19, Tata Inst. Fund. Research, Bombay, 1960.

2. U. Cegrell, Removable singularity sets for analytic functions having modulus with bounded Laplace mass, Proc. Amer. Math. Soc. 88 (1983), 283-286.

3. J. A. Cima and I. R. Graham, On the extension of holomorphic functions with growth conditions across analytic subvarieties, Michigan Math. J. 28 (1981), 241-256.

4. K. Gowrisankaran, Multiply harmonic functions, Nagoya Math. J. 28 (1966), 27-48.

5. M. Heins, Hardy classes on Riemann surfaces, Lecture Notes in Math., vol. 98, Springer-Verlag. Berlin and New York, 1969.

6. P. Järvi, Removable singularities for $H^{p}$-functions, Proc. Amer. Math. Soc. 86 (1982), 596-598.

7. M. Parreau, Sur les moyennes des fonctions harmoniques et analytiques et la classification des surfaces de Riemann, Ann. Inst. Fourier (Grenoble) 3 (1951), 103-197.

8. W. Rudin, Function theory in polydiscs, Benjamin, New York, 1969.

9. D. Singman, Exceptional sets in a product of harmonic spaces, Math. Ann. 262 (1983), 29-43.

Department of Mathematics, University of Toronto, Toronto M5S 1A7, Ontario, Canada

Current address: Department of Mathematics, Pennsylvania State University, Mont Alto Campus, Mont Alto, Pennsylvania 17237 